\title{
Single-prolonged stress induces apoptosis in dorsal raphe nucleus in the rat model of posttraumatic stress disorder
}

\author{
Dongjuan Liu', ${ }^{1,2}$ Bing Xiao ${ }^{1}$, Fang Han², Enhua Wang ${ }^{3}$ and Yuxiu Shi ${ }^{1,2,3^{*}}$
}

\begin{abstract}
Introduction: Post-traumatic stress disorder (PTSD) is an anxiety disorder that develops after exposure to a life-threatening traumatic experience. Meta-analyses of the brainstem showed that midsagittal area of the pons was significantly reduced in patients with PTSD, suggesting a potential apoptosis in dorsal raphe nucleus after single-prolonged stress (SPS). The aim of this study is to investigate whether SPS induces apoptosis in dorsal raphe nucleus in PTSD rats, which may be a possible mechanism of reduced volume of pons and density of gray matter.

Methods: In this study, rats were randomly divided into $1 d, 7 d$ and $14 \mathrm{~d}$ groups after SPS along with the control group. The apoptosis rate was determined using annexin V-FITC/PI double-labeled flow cytometry (FCM). Levels of Cytochrome c (Cyt-C) was examined by Western blotting. Expression of Cyt-C on mitochondria in the dorsal raphe nucleus neuron was determined by enzymohistochemistry under transmission electron microscopy (TEM). The change of thiamine monophosphatase (TMP) levels was assessed by enzymohistochemistry under light microscope and TEM. Morphological changes of the ultrastructure of the dorsal raphe nucleus neuron were determined by TEM.
\end{abstract}

Results: Apoptotic morphological alterations were observed in dorsal raphe nucleus neuron for all SPS-stimulate groups of rats. The apoptosis rates were significantly increased in dorsal raphe nucleus neuron of SPS rats, along with increased release of cytochrome c from the mitochondria into the cytoplasm, increased expression of Cyt- $\mathrm{C}$ and TMP levels in the cytoplasm, which reached to the peak of increase 7 days of SPS.

Conclusions: The results indicate that SPS induced Cyt-C released from mitochondria into cytosol and apoptosis in dorsal raphe nucleus neuron of rats. Increased TMP in cytoplasm facilitated the clearance of apoptotic cells. We propose that this presents one of the mechanisms that lead to reduced volume of pons and gray matter associated with PTSD.

Keywords: Posttraumatic stress disorder, Dorsal raphe nucleus, Neuron, Apoptosis, Thiamine monophosphatase, Cytochrome c

\section{Background}

Post-traumatic stress disorder (PTSD) is a psychiatric condition that develops after being exposed to a lifethreatening traumatic experience. It is characterized with long-term symptoms of continuous experiences of the traumatic event, avoidance of stimuli associated with the

\footnotetext{
* Correspondence: shiyuxiu@163.com

'Department of Electron Microscopy, Basic Medical Sciences College, China Medical University, 92 North 2nd Road, Shenyang 110001Liaoning, China ${ }^{2}$ Department of Histology and Embryology, Basic Medical Sciences College, China Medical University, Shenyang, China

Full list of author information is available at the end of the article
}

trauma, numbing of general responsiveness, and increased arousal [1-3]. The pathophysiology of PTSD has been widely studied [4]. One of the core neuroendocrine abnormalities related to the disorder is the dysfunction of the hypothalamic-pituitary-adrenal (HPA) axis, characterized by low levels of adrenocorticotropic hormone (ACTH), plasma cortical and urinary cortisol and enhanced suppression of cortisol in response to low-dose dexamethasone administration [5,6]. Volumetric imaging studies have shown abnormal brain morphology in PTSD when compared with control subjects, and volumetric image

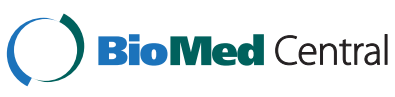


analysis of the brainstem showed smaller gray matter volume in the pons with PTSD [7]. However, the mechanism underline this finding has not been elucidated.

The dorsal raphe nucleus is located on the midline of the brainstem. It is a part of the raphe nucleus, locate at upper part of pons to caudal subdivisions of the oculomotor nucleus [8]. Dorsal raphe nucleus involves in multiple behavioral and physiological processes [9], and plays an important role in response to traumatic stimuli. In our previous study, we have reported that there is an increase of 5-HT1A in the dorsal raphe nucleus in the rats upon Single-prolonged stress (SPS) [10], which might be related to apoptosis in the dorsal raphe nucleus.

Apoptosis is a genetically programmed, morphologically distinct form of cell death that can be triggered by a variety of physiological and pathological stimuli [11]. There are distinct mechanisms that execute apoptosis according to various apoptotic stimuli. Apoptosis can be induced by two major pathways: the intrinsic pathway (mitochondria-dependent pathway) and the extrinsic pathway (death receptor-dependent pathway). Both of the pathways are regulated by caspases [12]. Caspases transduce the apoptotic signal cascade and engage the cellular targets that lead to programmed cell death $[13,14]$. In response to the apoptotic stimuli, mitochondria were impaired, resulting in the release of cytochorme $\mathrm{C}$ ( Cyt-C) from mitochondria into cytoplasm [15]. Cyt- $C$, a key enzyme in the respiratory chain, not only takes part in regulation of apoptosis but also is the key in mediating apoptosis upon being released into the cytosol. Cyt- $\mathrm{C}$ is also an intermediate in apoptosis, a controlled form of cell death used to kill cells in the process of development or in response to infection or DNA damage [16]. Thiamine monophosphatase (TMP), the hallmark of lysosomal functional state, is involved in the process of cell degeneration, necrosis and clearance of apoptotic cells $[17,18]$. SPS, which shows timedependent dysregulation in the HPA axis, is one of the widely used animal models for PTSD research [19].

In this study, in effort to elucidate the mechanisms underlying decreased volume of pons associated with PTSD, we employed the SPS model to investigate the changes of apoptosis rate, Cyt-C and TMP in dorsal raphe nucleus neurons as well as the morphological changes in ultrastructure of the dorsal raphe nucleus neuron by PTSD. Our data support the hypothesis that SPS-induced apoptosis in dorsal raphe nucleus contributes to the reduced volume of pons and density of gray matter in patients with PTSD.

\section{Materials and methods}

\section{Experimental animals}

Eight weeks-old Wistar rats (approximately 180 -200 g), provided by the Animal Experimental Center of China
Medical University, were used for all experiments. The rats were housed singly in clear polycarbonate cages $(46 \times 24 \times 20 \mathrm{~cm})$ for one week prior to the experiments. All rats were habituated to their cage and given standard food pellets and water. They were housed under a reversed the $12 \mathrm{~h}: 12 \mathrm{~h}$ light/ dark cycle (lights off at 10.00 a.m) and ambient temperature $\left(23 \pm 2^{\circ} \mathrm{C}\right)$ with humidity of $55 \pm 5 \%$. Rats were exposed to SPS on the day when weighed approximately between 230 and $250 \mathrm{~g}$. All animal protocols were carried out in accordance with the Guidance Suggestions for the Care and Use of Laboratory Animals formulated by the Ministry of Science and Technology of the People's Republic of China [20] and approved by Welfare \& Ethics Committee of Exprimental, China Medical University. All efforts were made to reduce the number of animals used and to minimize animal pain during the experiment.

\section{Model establishment and grouping}

After lab adaptation and handling, the rats were randomly assigned to one of the four groups and each group contains twenty rats. One group was served as a control group, while the others were SPS groups. The control rats were killed after the acclimation period, while the SPS groups rats were exposed to SPS procedure. The SPS model was prepared according to the previous report $[21,22]$. Briefly, each rat was restrained for two hours by placing it inside a clear disposable plastic bag with only the tail protruding. The plastic bag was closed with tape at the base of the tail. The size of plastic bags was adjusted according to the size of the rat in order to achieve complete immobilization. A hole in the plastic bags allowed the rats to breathe freely. After immobilization, rats were individually placed in a clear acrylic cylinder (240 $\mathrm{mm}$ in diameter and $500 \mathrm{~mm}$ height), and two thirds of the cylinder from the bottom was filled with water $\left(24^{\circ} \mathrm{C}\right)$. The rats were forced to swim for $20 \mathrm{~min}$. Following $15 \mathrm{~min}$ of recuperation, rats were kept undisturbed in their home cage. Consistent with time-dependent sensitization, behavioral experiments are generally undertaken $1 \sim 14$ days after the SPS procedure [23]. In this study, the SPS groups of animals were randomly assigned into three different groups depending on the days after SPS-stimulus: the group of 1 day after the SPS procedure $(n=20$; SPS $1 d$ group), the group of 7 days after the SPS procedure $(n=20$; SPS $7 d$ group), and the group of 14 days after the SPS procedure $(n=20$; SPS $14 d$ group). Each group included 20 rats, in which five rats were used for frozen sections, five for Western Blotting, five for flow cytometry, and five for TEM.

\section{Perfusion-based sections}

The rats of each group were anesthetized with 10\% chloral hydrate. The hearts were exposed, and the left 
ventricles were perfused with $200-300 \mathrm{~mL}$ of $0.9 \%$ saline via a catheter through the ascending aorta until a colorless infusion was achieved, followed by perfusion with a $300 \mathrm{~mL}$ of $4 \%$ paraformaldehyde (PFA) [24]. The whole brain were rapidly removed and dissected on ice, followed by $6-10 \mathrm{~h}$ of post-fixation in $4 \% \mathrm{PFA}$ at $4^{\circ} \mathrm{C}$. After being immersed in $20 \%$ sucrose solution and frozen in liquid nitrogen, coronal sections of the brain tissue were cut into slices of $12 \mu \mathrm{m}$ in thickness and stored at $-20^{\circ} \mathrm{C}$ for enzymohistochemistry morphological studies.

\section{Enzymohistochemistry analysis on expression of Cyt-C using TEM}

Sections of brain tissue in each group were washed with $0.1 \mathrm{M}$ phosphate-buffered saline (PBS) ( $\mathrm{pH}$ 7.4) for $30 \mathrm{~min}$ at room temperature, and incubated with incubation buffer containing (Cyt-C $10 \mathrm{mg}$, catalase $1 \mathrm{mg}, 0.2 \mathrm{M}$ PBS $5 \mathrm{ml}$, DAB $10 \mathrm{mg}$, distilled water $5 \mathrm{ml}$ ) at $4^{\circ} \mathrm{C}$ overnight. The samples were washed three times with PBS after incubation. To assess nonspecific staining, a few sections in every experimental group were incubated in PBS without incubation buffer. After washing, the samples were fixed with $1 \%$ osmium tetroxide at $4^{\circ} \mathrm{C}$ for $20 \mathrm{~min}$ and washed in PBS several times, dehydrated in gradient series $(20-100 \%)$ of ethanol and then in (100\%) acetone, infiltrated with Epon 812, and finally polymerized in pure Epon 812 at $65^{\circ} \mathrm{C}$ for $48 \mathrm{~h}$. The location of the dorsal raphe was determined on semi-thin sections. Ultra-thin sections $(70 \mathrm{~nm})$ were cut with ultramicrotome, collected on copper grids, and stained with $4 \%$ uranyl acetate. Five sections were selected from each group. Ten visual fields (about 250 cells) of the dorsal raphe nucleus in each section were examined with TEM (JEM-1200EX, Japan). The morphological changes and expression of Cyt- $\mathrm{C}$ of mitochondria were determined. The rates of change were calculated with the following formula: rate $=$ (numbers of mitochondria with morphological changes /total numbers of mitochondria in the cell) $\times 100 \%$.

\section{Western blotting analysis of Cyt-C expression}

Rats were anesthetized with $10 \%$ chloral hydrate and decapitated. The brain tissues were quickly removed and placed on ice. The dorsal raphe nucleus fragments were dissected from the brain tissues under a stereomicroscope in each experimental group according to the atlas of rats [25]. Proteins were extracted from the dorsal raphe nucleus samples of normal control rats or SPS rats through homogenization, ultrasonic dispersion and centrifugation. Extracted proteins were resuspended in the samples buffer containing $200 \mathrm{mM}$ tris-buffered saline (TBS), pH 7.5, 4\% sodium dodecyl sulfate (SDS), $20 \%$ glycerol, $10 \% \beta$-mercaptoethanol and boiled for $3 \mathrm{~min}$. The protein fraction $(50 \mu \mathrm{g} /$ lane $)$ prepared from each group was separated by $12 \%(\mathrm{w} / \mathrm{v})$ gradient sodium dodecyl sulfate (SDS)-polyacrylamide gel electrophoresis (PAGE) and transferred to a polyvinylidene fluoride (PVDF) membrane (Millipore, Bedford, MA, USA) using a semi-dry blotting apparatus (Bio-Rad Laboratories, Inc. Hercules, CA, USA). The membrane was blocked with 5\% dried skim milk, $0.05 \%$ Tween-20 in TBS at room temperature for $2 \mathrm{~h}$ and incubated with rabbit polyclonal antibody against Cyt-C IgG (Bipec Biopharma Corporation, U.S.A. $1: 200$ ) at $4^{\circ} \mathrm{C}$ for $2 \mathrm{~h}$. The membranes were washed three times with TBST (tris buffer with saline and tween 20), and then incubated with the anti-mouse IgG-HRP (Santa Cruz, U.S.A.; 1:5000) for $2 \mathrm{~h}$ at room temperature followed with TBST before visualization using enhanced chemiluminescence (ECL; Amersham Pharmacia Biotech, Buckinghamshire, UK). To confirm equal protein loading, the same blots were re-incubated with antibodies specific for $\beta$-actin (Abcam, British; 1:1,000) and detected with the ECL. The Optical Density (OD) was analyzed with the Gel Image Analysis System.

\section{Flow cytometry analysis of cell apoptosis rate in dorsal raphe nucleus}

Rats were anesthetized with $10 \%$ chloral hydrate and decapitated. The brain tissues were quickly removed and placed on ice. The dorsal raphe nucleus fragments were dissected from the brain tissues under a stereomicroscope in each experimental group according to the atlas of rats [25]. Single cell suspensions were then generated in cold PBS buffer, and the final concentrations were adjusted to $1 \times 10^{4} / \mathrm{ml}$. $1 \mathrm{ml}$ of suspensions were centrifuged it at $1000 \mathrm{rpm}$ for $10 \mathrm{~min}$ at $4^{\circ} \mathrm{C}$ and washed with $1 \mathrm{ml}$ cold PBS three times. Cell pellets were suspended in $200 \mu \mathrm{l}$ of binding buffer, and then incubated with $10 \mu \mathrm{l}$ of Annexin V-FITC and $5 \mu \mathrm{l}$ of Propidium Iodide at room temperature for $15 \mathrm{~min}$. Finally, $300 \mu \mathrm{l}$ of binding buffer was added into the incubation solution and was analyzed by flow cytometry (Becton Dicklnson, USA) in $1 \mathrm{~h}$. About $10^{4}$ of cells were analyzed by flow cytometry for each group. The apoptosis rate was calculated with the following formula: (number of apoptotic cells /total cells) $\times 100 \%$.

\section{Assessing the morphological change in dorsal raphe nucleus using TEM}

Dissected dorsal raphe nucleus samples were separated into $1 \mathrm{~mm}^{3}$ pieces, fixed with $2.5 \%$ glutaraldehyde at $4^{\circ} \mathrm{C}$. The pieces were post-fixed in $1 \%$ osmium tetroxide for $2 \mathrm{~h}$ at $4^{\circ} \mathrm{C}$, rinsed in $0.1 \mathrm{M}$ PBS $(\mathrm{pH} 7.4)$ several times, dehydrated in a gradient series (20-100\%) of ethanol and then in $100 \%$ acetone, infiltrated with Epon 812, and finally polymerized in pure Epon 812 at $65^{\circ} \mathrm{C}$ for $72 \mathrm{~h}$. The dorsal raphe nucleus was positioned in semithin sections. Ultra-thin sections $(70 \mathrm{~nm})$ were cut with 

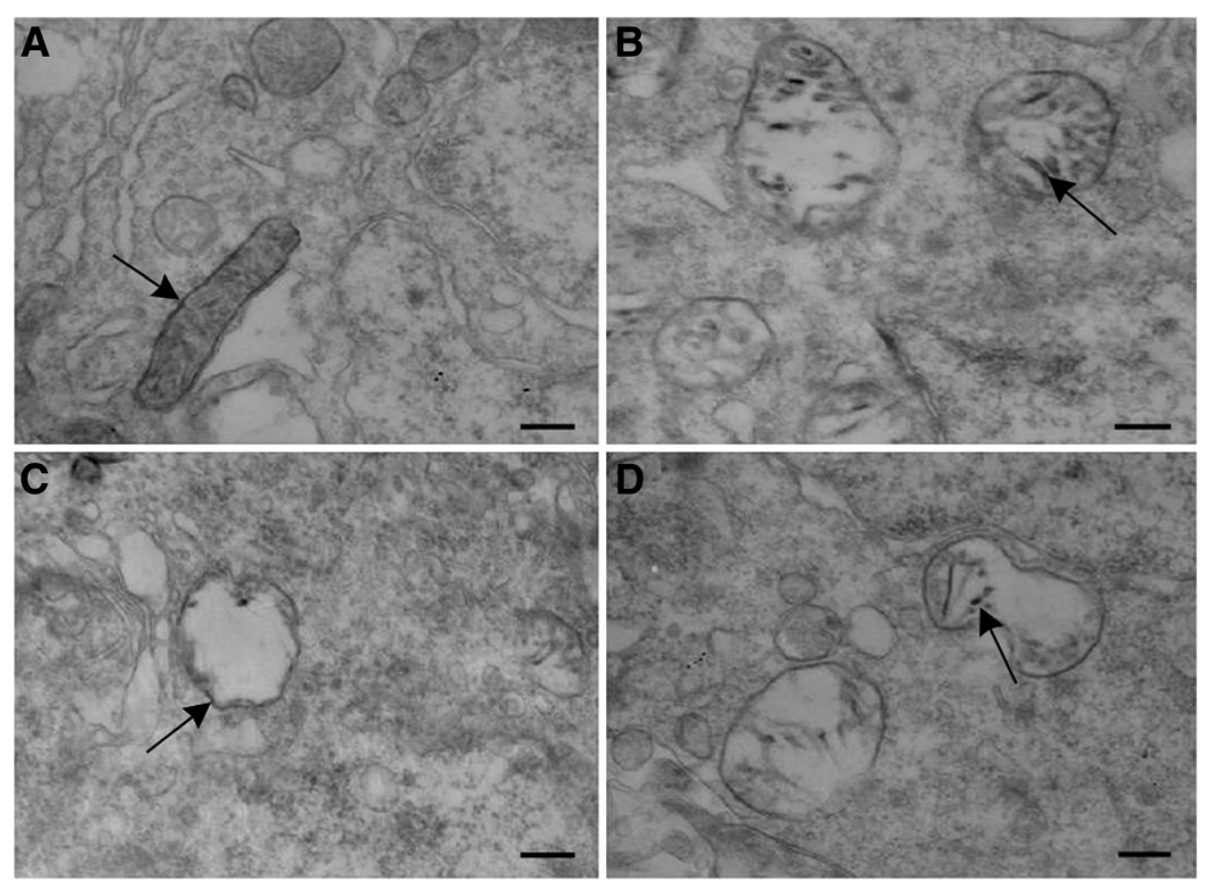

Figure 1 The distribution of $\mathrm{Cyt}-\mathrm{C}$ in mitochondria were found in dorsal raphe nucleus by TEM in each group (Figure 1A-D), Positive expression of $\mathrm{Cyt}-\mathrm{C}$, high electron-dense black granule $(\uparrow)$ in exterior and interior membrane of mitochondria. A: Mitochondria is intact, positive granule of Cyt-C were plentiful in control group; B: Part of Mitochondrial cristae fracture and membrane disruption,positive granule of Cyt-C were partly decreased in SPS1d group; C: Part of Mitochondrial vacuolization,positive granule of Cyt-C were obvious decreased in SPS 7d group. D: Part of Mitochondrial cristae fracture and membrane disruption,positive granule of Cyt-C were partly decreased in SPS14d group. All images are taken with identical magnification (Scale bar $=500 \mathrm{~nm}$ ).

ultramicrotome, collected on copper grids, stained with uranyl acetate and lead citrate. Five sections were selected from each group. Ten visual fields (about 250 cells) of the dorsal raphe nucleus in each section were examined with TEM (JEM-1200EX, Japan). The rates of the apoptotic cells was calculated with the following formula: rate $=$ (number of the apoptosis cells /total cells) $\times 100 \%$.

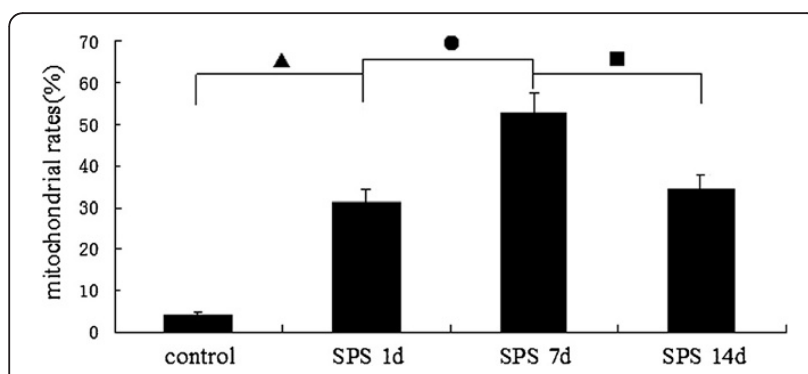

Figure 2 The rates of mitochondrial morphological change were calculated by TEM at different time points in dorsal raphe nucleus (mean \pm SEM). Most change was found at SPS $7 \mathrm{~d}$. $P<0.001$ SPS vs. control group. Tukey's test: $\boldsymbol{\Delta} P<0.05$ SPS $1 \mathrm{~d}$ vs. control group. $\bullet P<0.05$ SPS $7 d$ vs. SPS $1 d$. $P<0.05$ SPS $14 d$ vs. SPS $7 d$.

\section{Enzymohistochemistry analysis of TMP expression using light microscope}

Brain tissue sections in each group were washed with $0.1 \mathrm{M}$ phosphate-buffered saline (PBS) ( $\mathrm{pH}$ 7.4) for $30 \mathrm{~min}$ at room temperature, and samples were then incubated with TMP buffer [containing $15.29 \mathrm{~g}$ of TMP, $0.05 \mathrm{M}$ of acetic acid buffer $20 \mathrm{ml}$ (pH 3.9), $35.405 \mathrm{mg}$ of $\mathrm{CeCl}_{3}, 2.5 \mathrm{~g}$ of sucrose, $10 \mathrm{mg}$ of $\mathrm{DAB}, 30 \mathrm{ml}$ of distilled water, $0.000075 \mathrm{ml}$ of TritonX-100] for $80 \mathrm{~min}$ at $37^{\circ} \mathrm{C}$. To assess nonspecific staining, a few sections in every experimental group were incubated in PBS without incubating buffer. The sections were washed three times with PBS after incubation. Finally, 1\% Vulcanization amine

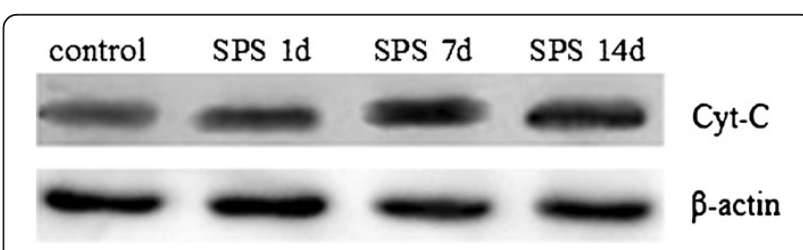

Figure 3 Western Blotting showed that the protein expression of Cyt-C increased in dorsal raphe nucleus of SPS rats compared with control levels. Protein expression of $C y t-C$ peaked at SPS $7 \mathrm{~d}$. 


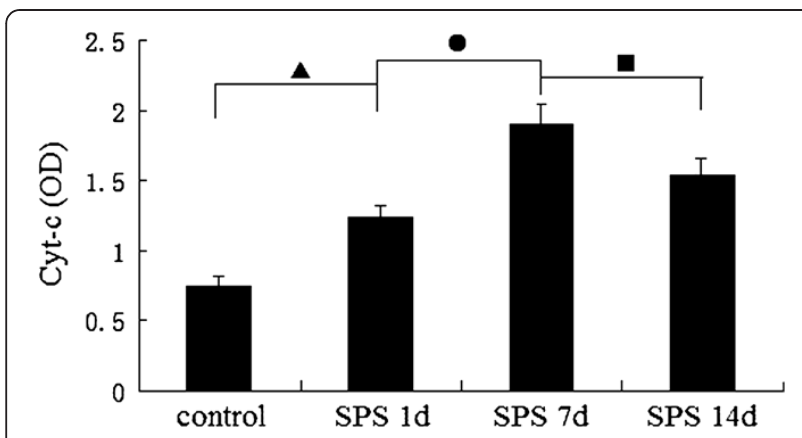

Figure 4 The mean optical densities of Cyt-C expression was shown at different time points in dorsal raphe nucleus, and peaked at SPS 7d (mean \pm SEM). $P<0.001$ SPS vs. control group. Tukey's test: $\boldsymbol{\Delta} P<0.05$ SPS $1 d$ vs. control group. $\bullet P<0.05$ SPS $7 d$ vs. SPS $1 d$. $P<0.05$ SPS $14 d$ vs. SPS $7 d$.

was used as chromogen for 1 min until the brown color appeared. Slides were then dehydrated and mounted with neutral gum. Five sections were selected from each group. Five visual fields of the dorsal raphe nucleus in each section were selected for examination $(\times 40)$. The optical density (OD) of TMP enzymohistochemistry positive cells in each field were analyzed using the MetaMorph/DPIO/ BX41 morphology image analysis system, and the average of OD were determined. The rates of the TMP positive enzymohistochemistry staining cells were calculated with the following formula: rate $=$ (number of the TMP positive cells/total cells) $\times 100 \%$.

\section{Enzymohistochemistry analysis of TMP expression using TEM}

After staining with $1 \%$ vulcanization amine as described above, the sections were washed with PBS. Then the sections were fixed in $1 \%$ osmium tetroxide for $20 \mathrm{~min}$ at $4^{\circ} \mathrm{C}$, rinsed in $0.1 \mathrm{M}$ PBS ( $\mathrm{pH}$ 7.4) several times,

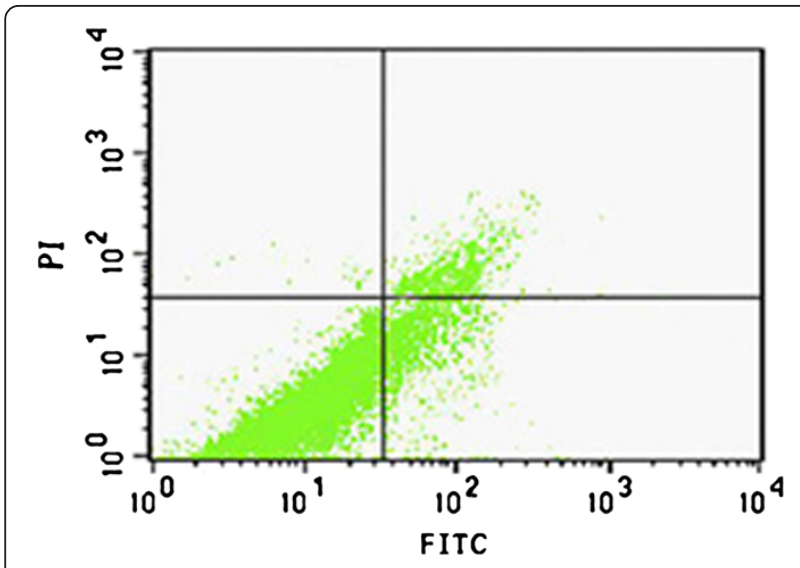

Figure 5 Flow cytometry detected the apoptosis rate in dorsal raphe nucleus at SPS7d. Flow cytometry pictographs: $X$-axis and $Y$-axis represents the relative intensity values of the fluorescence signal FITC and PI.

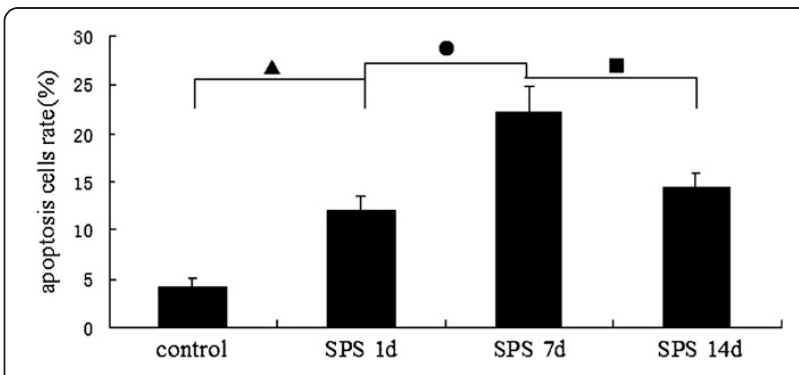

Figure 6 Double-labeled flow cytometry analyzed apoptosis rate in dorsal raphe nucleus at different time points (mean \pm SEM). The apoptosis rate peaked at SPS $7 \mathrm{~d}$. $P<0.001$ SPS vs. control group. Tukey's test: $\boldsymbol{\Delta} P<0.05$ SPS $1 \mathrm{~d}$ vs. control group. $\bullet P<0.05$ SPS 7d vs. SPS $1 d$. $\bullet<0.05$ SPS 14d vs. SPS 7d.

dehydrated in ethanol gradient $(20-100 \%)$ and then in $100 \%$ acetone, infiltrated with Epon 812, and finally polymerized in pure Epon 812 at $65^{\circ} \mathrm{C}$ for $48 \mathrm{~h}$. The dorsal raphe nucleus was positioned in the semi-thin sections. Ultra-thin sections $(70 \mathrm{~nm})$ were cut with ultramicrotome, collected on copper grids, and stained with uranyl acetate. Five sections were selected from each group. Ten visual fields (about 250 cells) of the dorsal raphe nucleus in each section were examined with TEM (JEM-1200EX, Japan). The rate of TMP-positive lysosome were calculated with the following formula: rate $=$ (number of positive expression of lysosome /total lysosome $) \times 100 \%$.

\section{Statistical analysis}

The results were expressed as mean \pm SEM. The differences between control group and the SPS groups were analyzed by one-way analysis of variance (ANOVA) using SPSS 13.0 software. In the case of significance with the ANOVA, post hoc comparisons were made using the Tukey's test to compare between groups. A level of $\mathrm{P}<0.05$ was considered to be statistically significant.

\section{Results}

\section{Enzymohistochemistry analysis of Cyt-C by TEM}

To investigate whether SPS induces apoptosis in dorsal raphe nucleus, we first examined Cyt-C expression by TEM. As shown in Figure 1, Cyt-C expression were detected and presented as high electron-dense black dots, which were distributed in the exterior and interior membranes of mitochondria. In contrast to the normal mitochondrial structure in control group (Figure 1A), many abnormal mitochondria were observed in SPS groups. Mitochondrial cristae fracture, vacuolization and membrane disruption were observed in samples from the group SPS 1d (Figure 1B), SPS 7d (Figure 1C) and SPS 14d (Figure 1D). In comparison to the control group, Cyt-C expression in the exterior and interior membranes of mitochondria were partly decreased in the SPS groups, with the translocation of Cyt-C from 

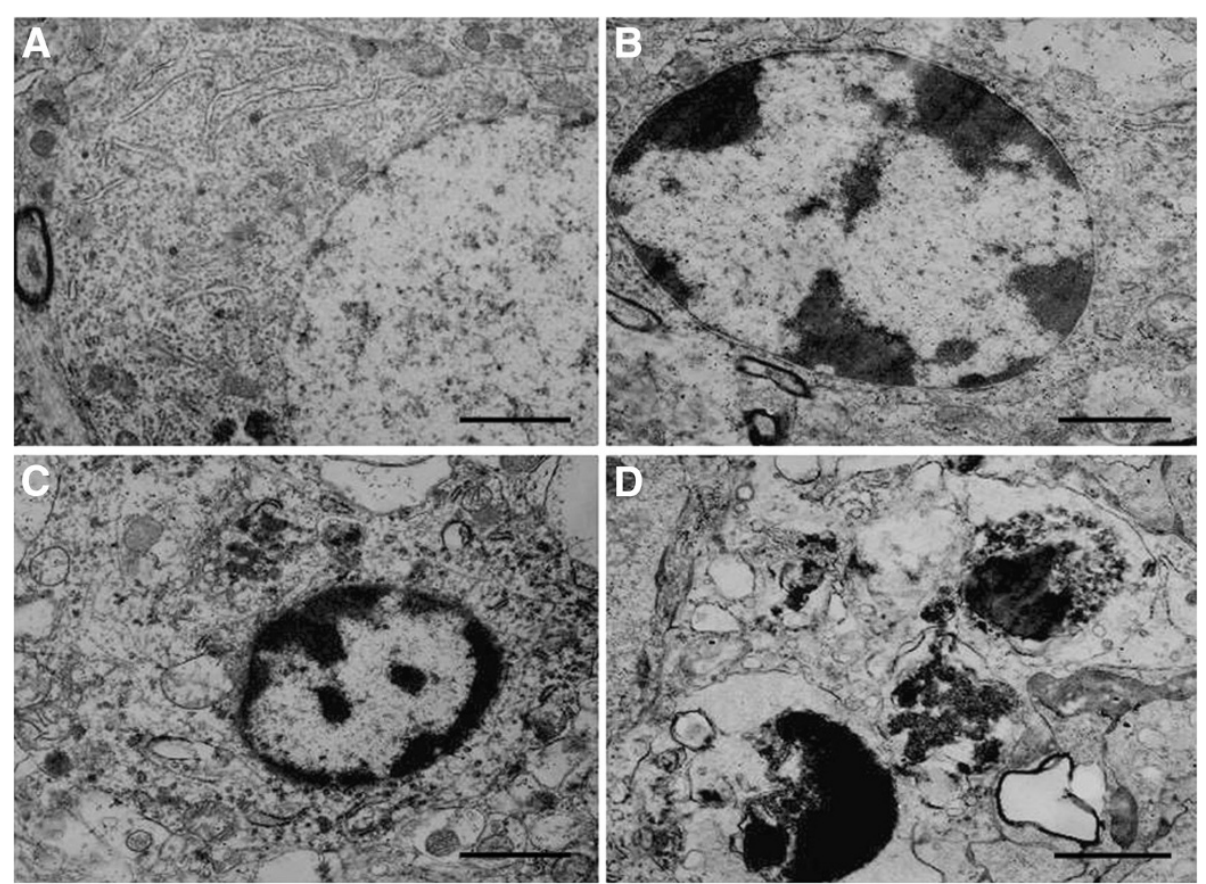

Figure 7 TEM images of the dorsal raphe nucleus region. A: control group; Dorsal raphe nucleus neuron had the nucleus is large, density of chromatin is uniform. B: SPS 1d group, Chromatin margination. C: SPS 14d group, Chromatin margination and appearance of crescent in chromatin. D: SPS 7d group,Appearance of apoptotic body, the cells showed some typical structural changes of apoptosis. All images are taken with identical magnification (Scale bar $=1 \mu \mathrm{m}$ ).

mitochondria to the cytoplasm of the cells in the SPS groups. Furthermore, this change was most obvious at SPS 7d. The rates of mitochondrial morphological change were reported in Figure 2 (Figure 2, $\mathrm{F}=184.741 \mathrm{df}=3,20$; $P<0.001$. Tukey's test $P<0.05)$.

\section{Western blotting analysis of Cyt-C}

We confirmed expression of Cyt-C by western blotting analysis. As shown in Figure 3, the Cyt-C and $\beta$-actin proteins were detected at 17 and $42 \mathrm{kDa}$, respectively, and the mean values of the band densities of the control group were set as $100 \%$. The result showed that Cyt-C

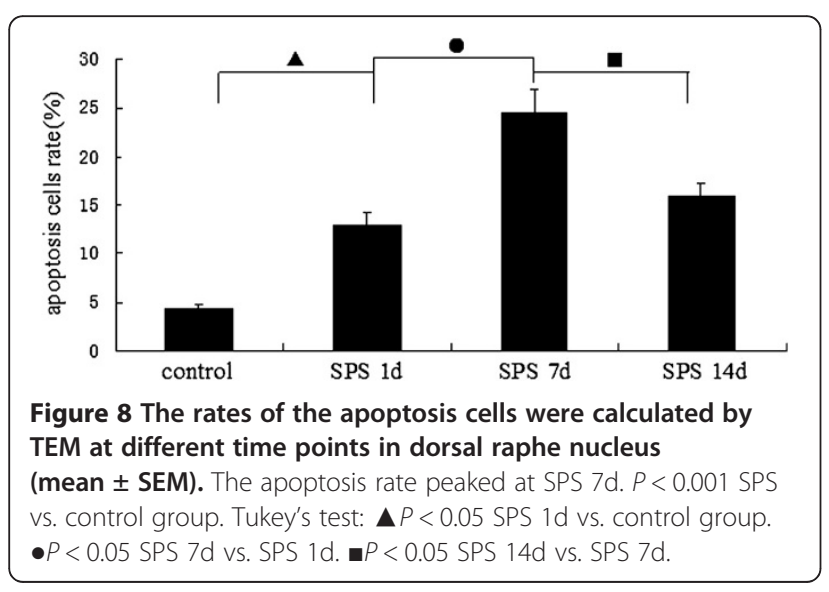

expressions in the SPS groups were significantly increased in comparision to that of the control group. The peak of increase of Cyt-C levels occurred in SPS 7d, but gradually decreased at SPS $14 \mathrm{~d}$. The mean optical densities of Cyt-C expression was indicated in Figure 4 (Figure 4, $\mathrm{F}=98.829 \mathrm{df}=3,20 ; P<0.001$. Tukey's test $P<0.05)$.

\section{Analysis apoptosis rate by flow cytometry}

We further determined the numbers of apoptotic cells upon SPS by flow cytometry using Annexin V-FITC /PI staining. Rats in the SPS $7 d$ group had considerable amounts of apoptotic cells in dorsal raphe nucleus cells (Figure 5). These cells were in cluster-shape distribution, with viable cell population (Annexin $\mathrm{V}^{-} / \mathrm{PI}^{-}$) distributed in the left lower quadrant region, early apoptotic cell population (Annexin $\mathrm{V}^{+} / \mathrm{PI}^{-}$) in the right lower quadrant region, late apoptotic cell populations $\left(A n n e x i n \mathrm{~V}^{+} / \mathrm{PI}^{+}\right.$) in the right upper quadrant region, and the necrotic cell population (Annexin $\mathrm{V}^{-} / \mathrm{PI}^{+}$) in the upper left quadrant region. In comparison to the control group, SPSstimulus group showed increased apoptotic cell populations in dorsal raphe nucleus cells. The highest increase of apoptotic cells occurred in the SPS $7 \mathrm{~d}$ group. The apoptosis rate was reported in Figure 6 (Figure 6, $\mathrm{F}=99.322 \mathrm{df}=3,20 ; P<0.001$. Tukey's test $P<0.05)$. The rate began to decrease in the SPS $14 \mathrm{~d}$ group. These data 

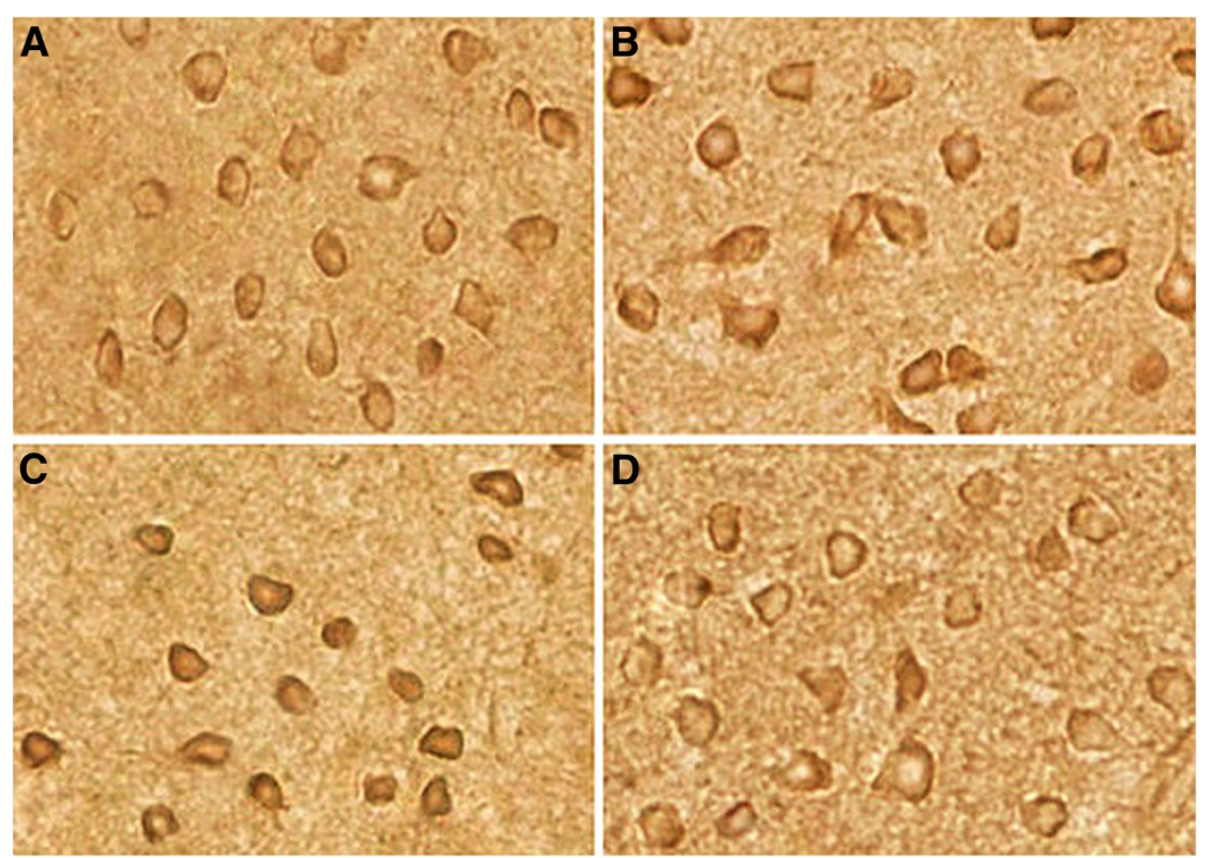

Figure 9 Expression of TMP in dorsal raphe nucleus was detected by enzymohistochemistry at different time points. A: control group; B: SPS1d group; C: SPS7d group; D: SPS14d group. All images are taken with identical magnification (x 400).

demonstrate that SPS induces apoptosis in dorsal raphe nucleus cells.

\section{Morphological change of the dorsal raphe nucleus by TEM}

As shown in Figure 7, we further performed TEM to assess the morphological changes of the dorsal raphe nucleus upon SPS stimulus. In control rats, dorsal raphe nucleus cells exhibited a normal structure (Figure 7A). However, upon SPS stimulus, the nucleus showed variable degree of structural alterations. A portion of cells in SPS stimuli rats had characteristic of apoptosis, with chromatin condensation and margination (Figure 7B,C),

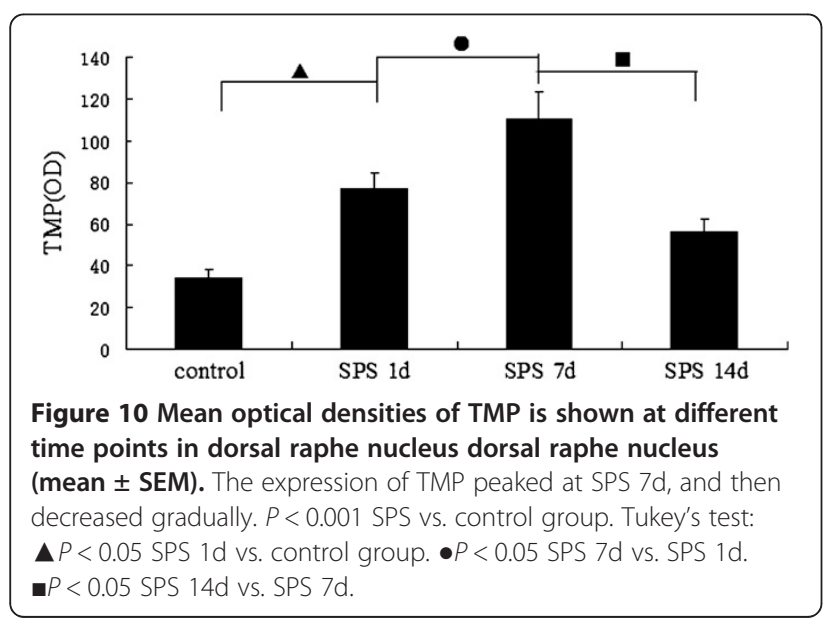

appearance of chromatin crescents (Figure 7C) and apoptotic body (Figure 7D). Furthermore, these changes were found mostly in group SPS 7d. Quantitative analysis of the apoptotic changes was reported in Figure 8 (Figure $8, \mathrm{~F}=146.252 \mathrm{df}=3,20 ; P<0.001$. Tukey's test $P<0.05)$.

\section{Enzymohistochemistry analysis of TMP by light microscope and TEM}

We examined the distribution of thiamine monophosphatase (TMP) in samples from SPS- stimulus or control rats. Enzymohistochemistry staining analysis showed that TMP were widely distributed throughout the dorsal

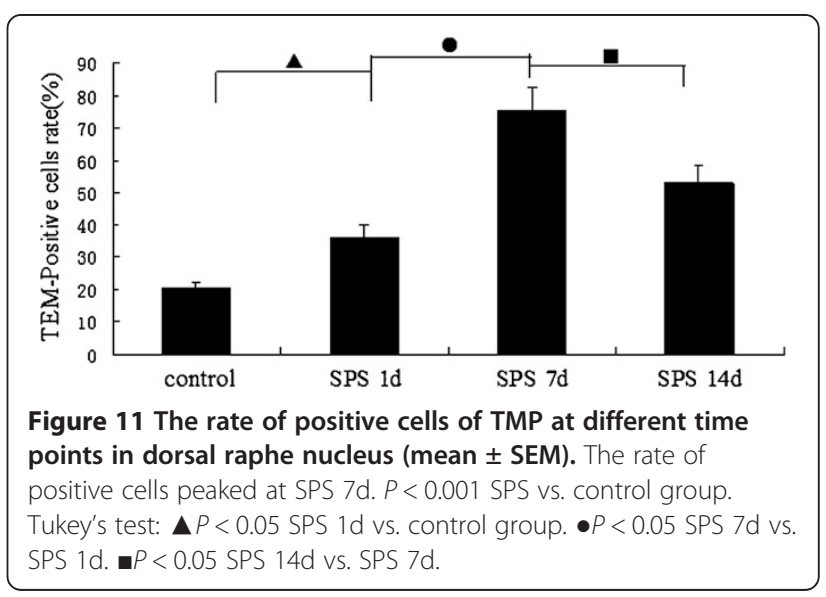



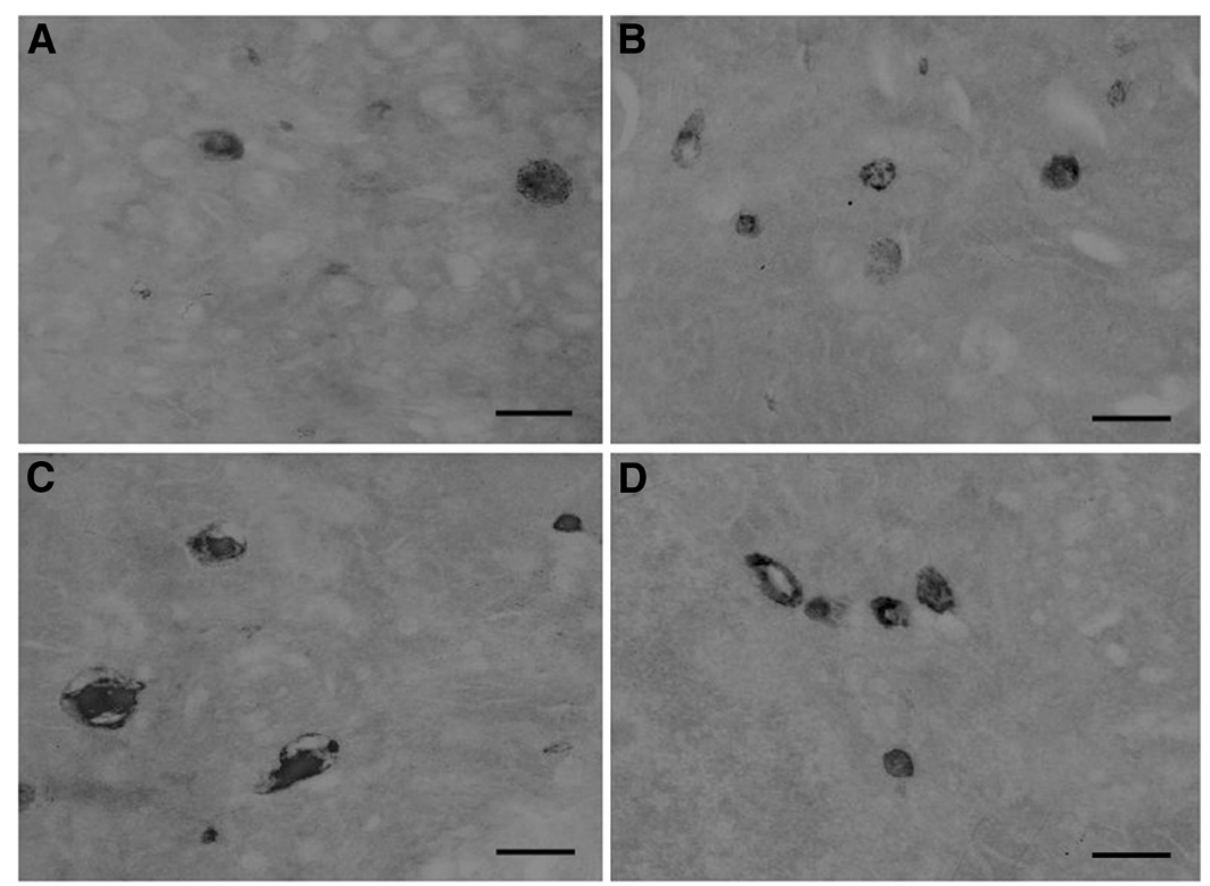

Figure 12 The distribution of TMP in lysosome were found in dorsal raphe nucleus by TEM in each group, Positive expression of TMP produces high electron-dense black granule( $\uparrow$ ). A: control group; B: SPS1d group; C: SPS7d group; D: SPS14d group. The membrane disruption of lysosomes and the translocation of TMP from lysosome to cytoplasm appeared in SPS group. All images are taken with identical magnification (Scale bar $=500 \mathrm{~nm}$ ).

raphe nucleus neuron region, mainly in the cytoplasm (TMP-positive cells were stained in brown, Figure 9). There was a significant increase of TMP content in the SPS groups compared to the control group (Figure 9A-D). The increase in TMP expression peaked in group SPS 7d, and TMP expression began decrease in group SPS 14d. The mean optical densities of TMP is shown in Figure 10 (Figure 10, $\mathrm{F}=79.623 \mathrm{df}=3,20 ; P<0.001$. Tukey's test $P<0.05)$. The rate of positive cells of TMP was reported in Figure 11 (Figure 11, $\mathrm{F}=119.084 \mathrm{df}=3,20 ; P<0.001$. Tukey's test $P<0.05)$.

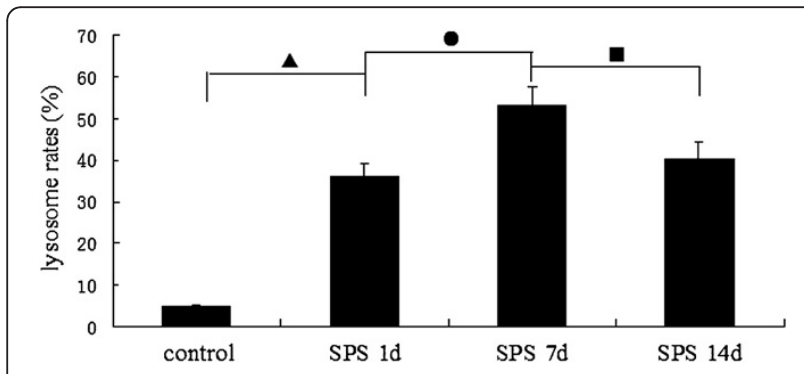

Figure 13 The rates of lysosome which have positive expression of TMP were calculated by TEM at different time points in dorsal raphe nucleus (mean $\pm \mathrm{SEM}$ ). This change was found mostly at SPS 7d. $P<0.001$ SPS vs. control group. Tukey's test: $\Delta P<0.05$ SPS 1 d vs. control group. $\bullet P<0.05$ SPS $7 d$ vs. SPS $1 d$. a $P<0.05$ SPS $14 \mathrm{~d}$ vs. SPS $7 \mathrm{~d}$.
Levels of TMP expression were also evaluated using TEM method. As shown in the Figure 12, positive TMP expression results in high electron-dense black granules that were located in the lysosomes in the dorsal raphe nucleus neuron (Figure 12A-D). Intracellular lysosomes had a normal round-shape structure in control group (Figure 12A). However, many lysosomes with abnormal shape were observed in SPS groups (Figure 12B,D). The membrane disruption of lysosomes and the translocation of TMP from lysosome to cytoplasm were also observed in SPS groups(Figure 12B,C). The numbers of lysosomes with positive expression of TMP were significantly increased in the SPS groups compared to the control group. Again, the most dramatic change occurred in group SPS 7d. The rates of lysosome which have positive expression of TMP was reported in Figure 13 (Figure 13, $\mathrm{F}=187.044 \mathrm{df}=3,20 ; P<0.001$. Tukey's test $P<0.05)$.

\section{Discussion}

PTSD is a disfunction in response to fear-related stimuli. Four major types of characteristic symptoms associated with PTSD are: re-experiencing, avoidance, numbing and hyperarousal [1]. Single-prolonged stress (SPS), a well established animal model for studying PTSD [26], induces enhanced inhibition of the hypothalamicpituitary-adrenal (HPA) axis, which has been found in patients with PTSD. SPS-stimulus rats also exhibit 
behavioral abnormalities (enhanced anxiety) that mimic the symptoms associated with PTSD patients [19,21,27]. The dorsal raphe nucleus involves in many physiological activities, and its function has been associated to PTSD. Several lines of evidence have implicated the dorsal raphe nucleus as a main substrate for modulation of nocuous stress. Volumetric image analysis of the brainstem showed smaller gray matter volume in the pons with PTSD [7], which might be related to apoptosis. Thus, this study focused on the changes of the dorsal raphe nucleus in rat after SPS stimulus, and determined whether apoptosis occurs in the dorsal raphe nucleus after exposure to SPS.

Apoptosis is a genetically controlled, complex process that is central to normal development, homeostasis and disease development. Apoptosis is activated in response to environmental signals or being triggered by intrinsic factors. The process is designed to kill and remove errant or aging cells [28]. Apoptosis of neurons involves many cellular and molecular processes. In this study, we determined the apoptosis rate in the dorsal raphe nucleus using Annexin V-FITC/PI double-labeled flow cytometry (FCM). The results showed that SPS stimuli induced apoptosis of dorsal raphe nucleus neuron and the rate of apoptosis peaked at day 7 after SPS stimuli. We observed morphologic changes characteristic of apoptosis in the dorsal raphe nucleus neuron [29], such as chromatin condensation, chromatin margination, chromatin crescents, nucleus fragmentation, nucleus disappearance and apoptotic body.

Mitochondria play a central role in cellular survival and apoptotic death [30,31]. Mitochondria participate in apoptosis by transduction or magnification of apoptotic signal. Cyt-C, a key protein in electron transport, is located on mitochondrial inner and outer membrane [32], and also plays a key role in apoptosis induced by the mitochondrial pathway [33]. Our study indicates that Cyt- $\mathrm{C}$ were released from mitochondria to cytoplasm in dorsal raphe nucleus neurons after exposure to SPS. In cytoplasm, released of Cyt-C was appear in SPS 1d group and peaked in SPS $7 \mathrm{~d}$ group. Numbers of apoptotic cells in dorsal raphe nucleus peaked at SPS $7 \mathrm{~d}$, determined by Annexin V-FITC/ PI double-labeled FCM analysis. These results demonstrated that the release of Cyt-C occured earlier than that of the apoptotic peak. These results support the notion that the mitochondrial signaling pathway is a critical process of apoptosis in dorsal raphe nucleus in the rat model of PTSD. We also examined the effect of SPS on activity of Cyt-C by Western blotting and demonstrated that the levels of Cyt-C increased upon SPS treatment.

Several signals can trigger apoptosis via different pathways, involving a variety of enzymatic reactions, including proteolytic enzymes from lysosome. TMP protease is the hallmark enzyme of lysosome, and its activity represents the status of lysosomal function. Sheen et al. reported that apoptosis increases the permeability of lysosome and leads to the release of proteases and protein kinases [17]. In this study, we measured the TMP levels in the dorsal raphe nucleus using enzymohistochemistry. We found that TMP activity was upregulated and TMP enzymes were released from lysosome upon SPS stimuli, with the highest change occurred in SPS 7d group. Thus, SPS leads to the dysfunction of lysosome of dorsal raphe nucleus, increase of permeability and rupture of lysosomal membrane. This further leads to TMP released from lysosome to cytoplasm. Increased TMP in cytoplasm facilitates the degradation, dispose and clearance of apoptotic cells products. Thus, apoptosis and overexpression of TMP may be one of the pathophysiological foundation for the behavior disorder in PTSD.

\section{Conclusion}

Our results indicate that SPS induces Cyt-C release, apoptosis, and increased TMP in dorsal raphe nucleus neuron in rats. This may result in reduced volume of the dorsal raphe nucleus. This may present a possible mechanism that leads to reduced volume of pons and gray matter associated with PTSD.

\section{Abbreviations}

ACTH: Adrenocorticotropic hormone; ANOVA: Analysis of variance; CytC: Cytochrome c; ECL: Enhanced chemiluminescence; HPA: Hypothalamicpituitary-adrenal; OD: Optical density; PAGE: Polyacrylamide gel electrophoresis; PBS: Phosphate-buffered saline; P FA: Paraformaldehyde; PTSD: Post-traumatic stress disorder; PVDF: Polyvinylidene fluoride; SDS: Sodium dodecyl sulfate; SPS: Single-prolonged stress; TBS: Tris-buffered saline; TBST: Tris buffered saline tween; TEM: Transmission electron microscopy; TMP: Thiamine monophosphatase.

\section{Competing interests}

The authors declare that they have no competing interests.

\section{Authors' contributions}

Dongjuan Liu conceived of the study and drafted the manuscript, all authors participated in the design and coordination of the study, and Bing Xiao performed the statistical analysis. All authors read and approved the final manuscript.

\section{Acknowledgments}

This research was supported by a grant from the National Natural Science Foundation of China (NO. 31140060 and 81171282). The authors would like to thank the anonymous reviewers for their valuable comments on how to improve the quality of the paper.

\section{Author details}

'Department of Electron Microscopy, Basic Medical Sciences College, China Medical University, 92 North 2nd Road, Shenyang 110001Liaoning, China. ${ }^{2}$ Department of Histology and Embryology, Basic Medical Sciences College, China Medical University, Shenyang, China. Institute of pathology and pathophysiology, China Medical University, Shenyang, China.

Received: 10 January 2012 Accepted: 15 November 2012

Published: 27 November 2012 


\section{References}

1. American Psychiatric Association: Diagnostic and Statistical Manual of Menta Disorders, 4th ed. DSM-IV. Washington, DC: American Psychiatric Press; 1994

2. Li S, Murakami Y, Wang M, Maeda K, Matsumoto K: The effects of chronic valproate and diazepam in a mouse model of posttraumatic stress disorder. Pharmacol Biochem Behav 2006, 85(2):324-331.

3. Siegmund A, Wotjak CT: A mouse model of posttraumatic stress disorder that distinguishes between conditioned and sensitised fear. J Psychiatr Res 2007, 41(10):848-860.

4. Kessler RC: Posttraumatic stress disorder: the burden to the individual and to society. J Clin Psychiatry 2000, 61(suppl 5):4-12.

5. Yehuda R, Golier JA, Halligan SL, Meaney M, Bierer LM: The ACTH response to Dexamethasone in PTSD. Am J Psychiatry 2004, 161(8):397-1403.

6. de Kloet CS, Vermetten E, Geuze E, Kavelaars A, Heijnen CJ, Westenberg HG: Assessment of HPA-axis function in posttraumatic stress disorder: pharmacological and non-pharmacological challenge tests, a review. J Psychiatr Res 2006, 40(6):550-567.

7. Carrion VG, Weems CF, Watson C, Eliez S, Menon V, Reiss AL: Converging evidence for abnormalities of the prefrontal cortex and evaluation of midsagittal structures in pediatric posttraumatic stress disorder: an MRI study. Psychiatry Res Neuroimaging 2009, 172(3):226-234.

8. O'Hearn E, Molliver ME: Organization of raphe-cortical projections in rat: a quantitative retrograde study. Brain Res Bull 1984, 13:709-726.

9. Morin $\mathrm{P}$, Meyer-Bernstein EL: The ascending serotonergic system in the hamster: comparison with projections of the dorsal and median raphe nuclei. Neuroscience 1999, 91:81-105.

10. Luo FF, Han F, Shi YX: Change in 5-HT1A receptor in the dorsal raphe nucleus in a rat model of post-traumatic stress disorder. Mol Med Rep 2011, 4:843-847.

11. Roulston A, Marcellus RC, Branton PE: Virus and apoptosis. Annu Rev Microbiol 1999, 53:577-628.

12. Thornberry NA, Lazebnik Y: Caspases: enemies within. Science 1998 , 281:1312-1316.

13. Peter ME, Krammer PH: The CD95(APO-1/Fas) DISC and beyond. Cell Death Differ 2003, 10(1):26-35.

14. Bantseev V, Youn HY: Mitochondrial "movement" and lens optics following oxidative stress from UV-B irradiation: cultured bovine lenses and human retinal pigment epithelial cells (ARPE-19) as examples. Ann N Y Acad Sci 2006, 1091:17-33.

15. Li P, Nijhawan D, Budihardjo I, Srinivasula SM, Ahmad M, Alnemri ES, Wang $X$ : Cytochrome $c$ and dATP-dependent formation of Apaf-1/caspase-9 complex initiates an apoptotic protease cascade. Cell 1997, 91:479-489.

16. Liu X, Kim C, Yang J, Jemmerson R, Wang X: Induction of apoptotic program in cell-free extracts: requirement for dATP and cytochrome $c$. Cell 1996, 86(1):147-157.

17. Doty SB, Smith CE, Hand AR, Oliver C: Inorganic trimetaphosphatase as a histochemical marker for lysosomes in light and electron microscopy. J Histochem Cytochem 1977, 25:1381-1384.

18. Sheen VL, Macklis JD: Apoptotic mechanisms in targeted neuronal cell ddeach by chromophore-activated photolysis. Exp Neurol 1994, 130(1):67-81.

19. Iwamoto Y, Morinobu S, Takahashi T, Yamawaki S: Single prolonged stress increases contextual freezing and the expression of glycine transporter 1 and vesicle-associated membrane protein 2 mRNA in the hippocampus of rats. Prog Neuropsychopharmacol Biol Psychiatry 2007, 31(3):642-651.

20. The Minixtry of Science and Technology of the People's Republic of China: Guidance Suggestions for the Care and Use of Labortory Animals; 2006.

21. Takahashi T, Morinobu S, Iwamoto Y, Yamawaki S: Effect of paroxetine on enhanced contextual fear induced by single prolonged stress in rats. Psychopharmacology (Berl) 2006, 189(2):165-173.

22. Kohda K, Harada K, Kato K, Hoshino A, Motohashi J, Yamaji T, Morinobu S, Matsuoka N, Kato N: Glucocorticoid receptor activation is involved in producing abnormal phenotypes of single-prolonged stress rats: a putative post-traumatic stress disorder model. Neuroscience 2007, 148(1):22-33.

23. Yamamoto S, Morinobu S, Takei S, Fuchikami M, Matsuki A, Yamawaki S, Liberzon I, et al: Single prolonged stress: toward an animal model of posttraumatic stress disorder. Depress Anxiety 2009, 26:1110-1117.

24. Liu HY: Technical operations and its common problems of perfusion fixation in mice. Qiqihaer Yixueyuan Xuebao 2006, 27(11):1341.
25. Paxinos G, Watson C: The Rat Brain in Stereotaxic Coordinates. Fourth Edition: Academic Press; 1998.

26. Liberzon I, Krstov M, Young EA: Stress-restress: effects on ACTH and fast feedback. Psychoneuroendocrino 1997, 22:443-453.

27. Imanaka A, Morinobu S, Toki S, Yamawaki S: Importance of early environment in the development of post-traumatic stress disorder-like behaviors. Behav Brain Res 2006, 173(1):129-137.

28. Yan Q, Liu JP, Li DW: Apoptosis in lens development and pathology. Differentiation 2006, 74(5):195-211.

29. Thomp son CB: Apoptosis in the pathogenesis and treatment of diseade. Science 1995, 267:1452

30. Finkel E: The mitochondrion: is it central to apoptosis? Science 2001, 292(5517):624-626.

31. Brenner C, Kroemer G: Apoptosis: mitochondria-the death signal integrators. Science 2000, 289(5482):1150-1151.

32. Tamilselvan J, Jayaraman G, Sivarajan K, Panneerselvam C: Ags-dependent upregulation of p53 and cytochrome $\mathrm{c}$ release and susceptibility to apoptosis in skeletal muscle fiber of aged rats: role of carnitine and lipoic acid. Free Radic Biol Med 2007, 43(12):1656-1669.

33. Latta M, Künstle $G$, Leist $M$, Wendel A: Metabolic depletion of ATP by fructose inversely controls CD95- and tumor necrosis factor receptor1-mediated hepatic apoptosis. J Exp Med 2000, 191(11):1975-1985

doi:10.1186/1471-244X-12-211

Cite this article as: Liu et al.: Single-prolonged stress induces apoptosis in dorsal raphe nucleus in the rat model of posttraumatic stress disorder. BMC Psychiatry 2012 12:211

\section{Submit your next manuscript to BioMed Central and take full advantage of:}

- Convenient online submission

- Thorough peer review

- No space constraints or color figure charges

- Immediate publication on acceptance

- Inclusion in PubMed, CAS, Scopus and Google Scholar

- Research which is freely available for redistribution 